\title{
Prevalence and Risk Factors of Hypertension in Hemodialysis
}

\author{
Imen Gorsane, Madiha Mahfoudhi*, Fathi Younsi, Imed Helal, Taieb Ben Abdallah \\ Internal Medicine A Department, Charles Nicolle Hospital, Tunis, Tunisia \\ Email: ${ }^{*}$ madiha mahfoudhi@yahoo.fr
}

Received 31 March 2015; accepted 13 June 2015; published 16 June 2015

Copyright (C) 2015 by authors and Scientific Research Publishing Inc.

This work is licensed under the Creative Commons Attribution International License (CC BY).

http://creativecommons.org/licenses/by/4.0/

\section{(c) (i) Open Access}

\section{Abstract}

The prevalence of hypertension in iterative hemodialysis (HD) remains high and was associated with a high morbidity and mortality. It was a single-center retrospective study including 124 patients on chronic HD in our unit. The prevalence of hypertension was determined from blood pressure (BP) monitoring in beginning, middle and end of dialysis. We defined hypertension as systolic BP (SBP) greater than or equal to $140 \mathrm{mmHg}$ and/or diastolic BP (DBP) greater than or equal to $90 \mathrm{mmHg}$ on at least two measures. We have established a comparative study between the group of hypertensive dialysis and those not hypertensive. The prevalence of hypertension was $69.35 \%(86 / 124)$. The mean age was 57.15 years with a sex ratio of 1.2. Echocardiograms, performed in $64.5 \%$ of patients, showed a high prevalence of cardiac consequences of hypertension with left ventricular hypertrophy in $80 \%$ of patients and an average ejection fraction of $62 \%$. Diabetes, dialysis one session per week and the non-compliance with lifestyle and dietary rules were significantly associated with hypertension in HD in our study. The effect of HD on BP is dose-dependent. The reduction of BP allows a lower risk of cardiovascular (CV) events and mortality in hypertensive patients.

\section{Keywords}

Hemodialysis, Blood Pressure, Hypertension, Ultrafiltration, Volume Expansion

\section{Introduction}

Hemodialysis patients are at high risk for CV complications. This persists as their most common cause of death. Hypertension remains the most prevalent treatable risk factor in these patients [1]. Control of hypertension is important for reducing morbidity and mortality.

Hypertension is common in HD patients with a prevalence rate of approximately 90\%. Appropriate BP targets

\footnotetext{
${ }^{*}$ Corresponding author.
} 
for these patients remain uncertain. Studies have shown major gaps between recommended practice and realworld clinical performance in hemodialysis populations, including management and control of BP.

The measurement of BP is a simple and reproducible method. The National Kidney Foundation Kidney Disease Outcomes Quality Initiative (NKF-KDOQI) BP targets are pre-HD < 140/90 mm Hg, post-HD < 130/80 $\mathrm{mm} \mathrm{Hg}$ [2].

Volume overload is a primary factor contributing to hypertension, and attaining true dry weight remains a priority for nephrologists. More, a wide variety of pathophysiological mechanisms are involved.

\section{Material and Methods}

This was a retrospective study including 124 patients on chronic hemodialysis in our unit conducted in January 2014.

We excluded from the study, patients with advanced diseases such as cancer and New York Heart Association (NYHA) stage IV heart failure and those with average hemodialysis $<6$ months. Patients were on bicarbonate dialysis by synthetic membranes of various surfaces, and the duration of dialysis was 4 hours.

A blood pressure monitoring was performed in beginning, middle and end of dialysis as well as the heart rate. BP was taken in sitting position after 5 - 10 minutes of rest. Post-HD values were taken 5 - 10 minutes following dialysis.

The weight is measured at the entrance and exit of the session.

Demographic, clinical data and laboratory parameters were collected from patient's medical records.

Echocardiograms data not exceeding 6 months is collected.

Comparative study of different clinical and biological parameters between the group of hypertensive dialysis patients and those not hypertensive was conducted.

Baseline characteristics were described as means and standard deviations for continuous variables, and frequencies and proportions for categorical variables.

A study of the correlation was made between the various parameters by the statistical test CHI2. $p$ value $\leq$ 0.05 was regarded as significant.

The ethics committee had no objections against this study since it reflects our clinical work habits and did not include supplementary measures (biological or radiological examinations).

\section{Results}

The prevalence of hypertension was 69.35\% (86/124), and it was both systolic and diastolic in $90 \%$ of patients. The mean SBP was $170 \mathrm{mmHg}$. The mean age was 57.15 years [24 - 79 years] with a sex ratio of 1.21. Twenty patients (23.25\%) were smokers.

Forty six patients (53.48\%) were chronic HD with 3 sessions per week, 27 (31.39\%) had two sessions per week and 13 (15.11\%) had one session per week. The average hemodialysis was 48.65 months [6 - 300 months]. The interdialytic weight gain was $2.7 \mathrm{~kg}$. Significant residual renal diuresis persisted in 12 patients (13.95\%). Thirty nine patients were diabetics (45.34\%). The initial nephropathy was diabetic nephropathy in 33 cases (38.37\%), hypertensive nephropathy in 28 cases (32.55\%), chronic glomerulonephritis in 13 cases (15.11\%), chronic interstitial nephritis in 7 cases (8.13\%) and chronic kidney disease of unknown etiology in 5 cases (5.81\%).

Table 1 shows patient's epidemiological data.

Fifty-seven (66.27\%) of the patients said respect the life style and dietary rules.

Only four patients (4.65\%) were on ASE. Echocardiograms, performed in $64.5 \%$ of patients, showed left ventricular hypertrophy in $80 \%$ of patients and an average ejection fraction of $62 \%$.

Table 2 shows clinical and laboratory patient's features.

Sixty per cent of patients were taking antihypertensive treatment. The third of them (19.76\%) took three anti hypertensive drugs. Forty five (52.32\%) patients were on RAS-blocking drugs.

A comparative study of different clinical and biological parameters between the group of hypertensive dialysis patients and those not hypertensive (Table 3), found that diabetes, dialysis one session per week and the noncompliance with lifestyle and dietary rules were significantly associated with hypertension in $\operatorname{HD}(p=0.01,0.01$ and 0.005 respectively). 
Table 1. Epidemiological data.

\begin{tabular}{ccc}
\hline Characteristic & Duration of disease (years) & Value \\
\hline Age (years) & & 57.15 \\
Sex Ratio M/F & & 1.21 \\
Smoke & & $20(23.25 \%)$ \\
Average hemodialysis (months) & 19.2 & 48.65 \\
Diabetes & & $39(45.34 \%)$ \\
Cause of renal failure & 21.5 & \\
Diabetic nephropathy & 18.1 & $33(38.37 \%)$ \\
Hypertensive nephropathy & 10.6 & $28(32.55 \%)$ \\
Chronic glomerulonephritis & 17.8 & $13(15.11 \%)$ \\
Chronic Interstitial Nephritis & 9.4 & $7(8.13 \%)$ \\
Chronic kidney disease of unknown etiology & & $5(5.81 \%)$ \\
\hline
\end{tabular}

Table 2. Clinical and laboratory features.

\begin{tabular}{cc}
\hline Parameters & Value \\
\hline Pre-HD systolic BP (mm Hg) & 170 \\
Pre-HD diastolic BP (mm Hg) & 87 \\
Pre-HD mean arterial pressure (mm Hg) & 123 \\
Pre-HD mean heart rate (bpm) & 76 \\
Post-HD systolic BP (mm Hg) & 132 \\
Post-HD diastolic BP (mm Hg) & 70 \\
Post-HD mean arterial pressure (mmHg) & 101 \\
Post-HD mean heart rate (bpm) & 88 \\
PTH (pg/ml) & 400 \\
Hb (g/d) & 8.2 \\
Albumin (g/l) & 29.91 \\
Interdialytic weight gain (Kg) & 2.7 \\
Residual renal diuresis & $12(13.95 \%)$ \\
Patients respecting life style and dietary rules & 57 (66.27\%) \\
Erythropoiesis stimulating agents & $4(4.65 \%)$ \\
\hline
\end{tabular}

\section{Discussion}

Hypertension is present in up to $90 \%$ of end stage renal disease (ESRD) patients irrespective of the etiology of kidney disease [3]. Hypertension is recognized as an important modifiable risk factor for progression of chronic kidney disease (CKD) to ESRD and overall cardiovascular morbidity and mortality [4].

The prevalence of hypertension in HD patients is $80 \%$ - 90\% [5]. In our study, it was of the order of $70 \%$.

Elevated pre-HD SBP was associated with the occurrence of de novo cardiac failure, coronary artery disease and left ventricular hypertrophy (LVH), while actually lower SBP was associated with increased mortality [6]. But the reduction of BP in actively treated patients was associated with lower risks of all-cause and CV mortality [7].

Clinical guidelines-derived treatment goals represent the standards for providing evidence-based treatments 
Table 3. Comparison of patient's characteristics between two groups: hypertensive and not hypertensive patients.

\begin{tabular}{|c|c|c|c|c|c|}
\hline & $\begin{array}{l}\text { Hypertensive } \\
(\mathrm{n}=86)\end{array}$ & CI & $\begin{array}{l}\text { Not hypertensive } \\
\qquad(\mathrm{n}=38)\end{array}$ & CI & $p$ \\
\hline Age (years) & 57.15 & & 54.2 & & 0.1 \\
\hline Sex ratio $M / F$ & 1.21 & & 1.11 & & 0.1 \\
\hline Smoke & $20(23.25 \%)$ & {$[14.3 \%-32.2 \%]$} & $5(13.15 \%)$ & {$[2.4 \%-23.9 \%]$} & 0.5 \\
\hline $\begin{array}{c}\text { Respecting lifestyle and } \\
\text { dietary rules }\end{array}$ & $57(66.27 \%)$ & {$[56.3 \%-76.3 \%]$} & $32(84.21 \%)$ & {$[72.6 \%-95.8 \%]$} & 0.005 \\
\hline Diabetes & $39(45.34 \%)$ & [34.8\% - 55.9\%] & $10(26.31 \%)$ & {$[12.3 \%-40.3 \%]$} & 0.01 \\
\hline Average HD (months) & 48.65 & & 47.21 & & 0.5 \\
\hline Interdialytic weight gain (Kg) & 2.7 & & 2.65 & & 0.1 \\
\hline Residual renal diuresis & $12(13.95 \%)$ & {$[6.6 \%-21.3 \%]$} & $18(47.36 \%)$ & {$[31.5 \%-63.2 \%]$} & 0.29 \\
\hline HD one session & $13(15.11 \%)$ & {$[7.5 \%-22.7 \%]$} & $2(5.26 \%)$ & {$[-1.8 \%-12.4 \%]$} & 0.01 \\
\hline HD two sessions & 27 (31.39\%) & {$[21.6 \%-41.2 \%]$} & $8(21.05 \%)$ & {$[8.1 \%-34 \%]$} & 0.5 \\
\hline HD three sessions & $46(53.48 \%)$ & {$[42.9 \%-64 \%]$} & $28(19.15 \%)$ & {$[6.6 \%-31.7 \%]$} & 0.5 \\
\hline Parathyroid hormone (pg/ml) & 400 & & 458 & & 0.21 \\
\hline Serum Albumin (g/l) & 29.91 & & 30.02 & & 0.39 \\
\hline Hemoglobin (g/dl) & 8.2 & & 7.9 & & 0.1 \\
\hline $\begin{array}{c}\text { Erythropoiesis } \\
\text { stimulating agents }\end{array}$ & $4(4.65 \%)$ & {$[0.2 \%-9.1 \%]$} & $1(0.02 \%)$ & {$[-0.4 \%-0.5 \%]$} & 0.5 \\
\hline
\end{tabular}

CI: Confidence interval.

for dialysis patients. Although BP levels < 140/90 mmHg are recommended by current dialysis guidelines [8].

The optimal timing and method of BP measurement has yet to be defined. Compared to predialysis or postdialysis BP measurements, mean BP better correlates with echocardiographic LVH and with all-cause of mortality [9]-[11]. Other authors find that mean post-HD ambulatory BP correlated more strongly with left ventricular mass index (LVMI) than either mean systolic or diastolic BP [12] [13].

If ambulatory or home BP measurements are unavailable, median intradialytic midweek BP is used to define clinic BP [14].

Salt and water retention with excess extracellular fluid volume is frequent in hemodialysis patients. Overhydration is responsible for volume and pressure overload. Extracellular volume excess is an important factor in the pathogenesis of arterial hypertension, and control of volume status by ultrafiltration and achievement of dry weight is considered an essential therapeutical approach [15] [16]. Volume reduction is associated with arterial pressure reduction and with decreased LVH [17]. Reducing dietary salt intake is considered to be a fundamental intervention in this population [18] [19].

The percentage of interdialytic weight gain predicts increased pre-HD systolic BP and greater reduction in systolic BP from pre to post-HD. This is seen particularly in non-diabetics, younger patients, and those with greater estimated dry weight. We should be less aggressive with BP in older patients or those with diabetes [17].

Measurements by 24-hours ambulatory BP monitoring have shown that intensification of ultrafiltration may improve the control of hypertension in these patients [15]. But it increases the risks for arteriovenous fistula complications and CV events [20].

More frequent or longer dialysis is the ideal option for bettering BP control in HD patients. Frequent HD had significantly greater reductions in pre-HD systolic BP and number of antihypertensive medications used. A significant reduction in left ventricular (LV) mass is found with frequent HD [21] [22].

Nocturnal HD is another option suggested to improve outcomes in HD patients by offering increased dialysis time and reducing the large fluctuations in fluid shifts that occur with conventional HD. Nocturnal HD showed improvements in BP (systolic, diastolic, and mean arterial pressure) and LV mass index [23]. 
For our patients, we cannot ensure in the hospital 3 dialysis sessions for all. When weight gain is important, we extend the session at 5 hours. We insist on achievement of dry weight.

The RAAS has long been implicated in the etiology of hypertension in HD patients. It has been shown that ESRD patients have higher sympathetic nervous system activity. Abnormal autonomic sympathetic nervous activity can manifest as an absence of a nocturnal dip in BP. Nocturnal or diurnal dipping in BP is frequently absent in both CKD and ESRD populations and is associated with adverse outcomes [24].

Endothelial cell dysfunction involves disrupted balance of vasoconstrictors and vasodilators mediators, with as consequences, increased vasoconstriction.

Treatment with antihypertensive agents in ESRD patients was associated with improved CV events and mortality [25] [26]. The current recommendation is to employ a RAAS-blocking drug as the first-line agent in patients on HD [4]. In addition to their beneficial effect on BP, RAAS inhibitors improve LVH and pulse wave velocity. Additional antihypertensive agents are frequently needed for persistent hypertension, and calcium channel blockers and beta-blockers are some of the next recommended therapies [8].

Arterial stiffness is a pathogenetic process that occurs naturally with aging, but is accentuated in ESRD. The consequence is increased SBP and pulse pressure, which contribute to LVH.

Secondary hyperparathyroidism that accompanies CKD may contribute to the high prevalence of hypertension. Systolic and diastolic BP were significantly increased in subjects with elevated parathyroid hormone (PTH). Treatment with vitamin D significantly lowered cytosolic calcium, PTH, and mean BP [4].

More, ASE used to correct the anemia associated with ESRD are also suspected of causing increases in BP by increased sensitivity to angiotensin II and adrenergic stimuli [27]. Because of unavailability of ASE in our hospital, 4 patients only were on ASE.

It can be seen that delay in connection on the machine, needle insertion, anxiety about dialysis, are also likely to play a significant role in raising pre-HD BP [24].

\section{Conclusions}

Hypertension is highly prevalent in HD patients. It is associated with CV disease, the leading cause of mortality in HD patients.

Although clinical trial evidence of a target BP that improves mortality does not currently exist. The clinician is faced with a dilemma on how to evaluate blood pressure and treat this condition.

Management of hypertension in HD patients should include the establishment and maintenance of the appropriate dry weight and limitation of interdialytic sodium/fluid intake.

Pharmacologic therapy should include RAAS inhibitors as first-line agents.

We found in our study that hypertension risk factors in HD were: diabetes, dialysis one session per week and the non-compliance with lifestyle and dietary rules.

However, the two groups being compared were not homogenous. Further studies with larger numbers could be more conclusive.

\section{Conflict of Interest}

There are no conflicts of interest.

\section{References}

[1] Thompson, S., Hemmelgarn, B., Wiebe, N., Majumdar, S., Klarenbach, S., Jindal, K., et al. (2012) Clinical Decision Support to Improve Blood Pressure Control in Hemodialysis Patients: A Non Randomized Controlled Trial. Journal of Nephrology, 25, 944-953. http://dx.doi.org/10.5301/jn.5000238

[2] Gul, A., Miskulin, D., Gassman, J., Harford, A., Horowitz, B., Chen, J., et al. (2014) Design of the Blood Pressure Goals in Dialysis Pilot Study. American Journal of the Medical Sciences, 347, 125-130. http://dx.doi.org/10.1097/MAJ.0b013e31827daee5

[3] Agarwal, R., Nissenson, A., Battle, D., Coyne, D., Trout, J. and Warnock D. (2003) Prevalence, Treatment, and Control of Hypertension in Chronic Hemodialysis Patients in the United States. The American Journal of Medicine, 115, 291-297. http://dx.doi.org/10.1016/S0002-9343(03)00366-8

[4] Van Buren, P.N. and Inrig, J.K. (2012) Hypertension and Hemodialysis: Pathophysiology and Outcomesin Adult and Pediatric Populations. Pediatric Nephrology, 27, 339-350. http://dx.doi.org/10.1007/s00467-011-1775-3 
[5] Inrig, J., Oddone, E., Hasselblad, V., Gillespie, B., Patel, U.D., Reddan, D., et al. (2007) Association of Intradialytic Blood Pressure Changes with Hospitalization and Mortality Rates in Prevalent ESRD Patients. Kidney International, 71, 454-461. http://dx.doi.org/10.1038/sj.ki.5002077

[6] Foley, R., Parfrey, P., Darnett, J., Kent, G., Murray, D. and Barre, P. (1996) Impact of Hypertension on Cardiomyopathy, Morbidity, and Mortality in End-Stage Renal Disease. Kidney International, 49, 1379-1385. http://dx.doi.org/10.1038/ki.1996.194

[7] Heerspink, H.J., Ninomya, T., Zoungas, S., de Zeeuw, D., Grobbee, D.E., Jardine, M.J., et al. (2009) Effect of Lowering Blood Pressure on Cardiovascular Events and Mortality in Patients on Dialysis: A Systematic Review and MetaAnalysis of Randomised Controlled Trials. The Lancet, 373, 1009-1015. http://dx.doi.org/10.1016/S0140-6736(09)60212-9

[8] K/DOQI Workgroup (2005) K/DOQI Clinical Practice Guidelines for Cardiovascular Disease in Dialysis Patients. American Journal of Kidney Diseases, 45, S1-S153.

[9] Agarwal, R., Brim, N.J., Mahenthiran, J., Andersen, M.J. and Saha, C. (2006) Out-of-Hemodialysis-Unit Blood Pressure is a Superior Determinant of Left Ventricular Hypertrophy. Hypertension, 47, 62-68. http://dx.doi.org/10.1161/01.HYP.0000196279.29758.f4

[10] Inrig, J., Patel, U., Gillespie, B., Hasselblad, V., Himmelfarb, J., Reddan D., et al. (2007) Relationship between Interdialytic Weight Gain and Blood Pressure Among Prevalent Hemodialysis Patients. American Journal of Kidney Diseases, 50, 108.e4-118.e4. http://dx.doi.org/10.1053/j.ajkd.2007.04.020

[11] Agarwal, R. (2010) Blood Pressure and Mortality among Hemodialysis Patients. Hypertension, 55, 762-768. http://dx.doi.org/10.1161/HYPERTENSIONAHA.109.144899

[12] Harvey, P., Holt, A., Nicholas, J. and Dasgupta, I. (2013) Is an Average of Routine Postdialysis Blood Pressure a Good Indicator of Blood Pressure Control and Cardiovascular Risk? Journal of Nephrology, 26, 94-100. http://dx.doi.org/10.5301/jn.5000119

[13] Borsboom, H., Smans, L., Cramer, M.J., Kelder, J.C., Kooistra, M.P., Vos, P.F., et al. (2005) Long-Term Blood Pressure Monitoring and Echocardiographic Findings in Patients with End-Stage Renal Disease: Reverse Epidemiology Explained? Netherlands Journal of Medicine, 63, 399-406.

[14] Agarwal, R. (2012) The Controversies of Diagnosing and Treating Hypertension among Hemodialysis Patients. Seminars in Dialysis, 25, 370-376. http://dx.doi.org/10.1111/j.1525-139X.2012.01092.x

[15] Agarwal, R., Alborzi, P., Satyan, S. and Light, R.P. (2009) Dry-Weight Reduction in Hypertensive Hemodialysis Patients (DRIP): A Randomized, Controlled Trial. Hypertension, 53, 500-507. http://dx.doi.org/10.1161/HYPERTENSIONAHA.108.125674

[16] London, G.M. (2011) Ultrafiltration Intensification for Achievement of Dry Weight and Hypertension Control Is Not Always the Therapeutic Gold Standard. Journal of Nephrology, 24, 395-397. http://dx.doi.org/10.5301/jn.5000006

[17] Alborzi, P., Patel, N. and Agarwal, R. (2007) Home Blood Pressures Are of Greater Prognostic Value than Hemodialysis Unit Recordings. Clinical Journal of the American Society of Nephrology, 2, 1228-1234. http://dx.doi.org/10.2215/CJN.02250507

[18] Levin, N.W., Kotanko, P., Eckardt, K.U., Kasiske, B.L., Chazot, C., Cheung, A.K., et al. (2010) Blood Pressure in Chronic Kidney Disease Stage 5D-Report from a Kidney Disease: Improving Global Outcomes Controversies Conference. Kidney International, 77, 273-284. http://dx.doi.org/10.1038/ki.2009.469

[19] Charra, B. (2007) Fluid Balance, Dry Weight, and Blood Pressure in Dialysis. Hemodialysis International, 11, 21-31. http://dx.doi.org/10.1111/j.1542-4758.2007.00148.x

[20] Curatola, G., Bolignano, D., Rastelli, S., Caridi, G., Tripepi, R., Tripepi, G., et al. (2011) Ultrafiltration (UF) Intensification Improves Hypertension Control in Hemodialysis Patients but Increases Arterio-Venous Fistula Complications and Cardiovascular Events. Journal of Nephrology, 24, 465-473. http://dx.doi.org/10.5301/JN.2011.7735

[21] Zimmerman, D.L., Ruzicka, M., Hebert, P., Fergusson, D., Touyz, R.M. and Burns, K.D. (2014) Short Daily versus Conventional Hemodialysis for Hypertensive Patients: A Randomized Cross-Over Study. PLoS ONE, 9, e97135. http://dx.doi.org/10.1371/journal.pone.0097135

[22] Chertow, G.M., Levin, N.W., Beck, G.J., Depner, T.A., Eggers, P.W., et al., The FHN Trial Group (2010) In-Center Hemodialysis Six Times per Week versus Three Times per Week. New England Journal of Medicine, 363, $2287-2300$. http://dx.doi.org/10.1056/NEJMoa1001593

[23] Chan, C., Floras, J., Miller, J., Richardson, R. and Pierratos, A. (2002) Regression of Left Ventricular Hypertrophy after Conversion to Nocturnal Hemodialysis. Kidney International, 61, 2235-2239. http://dx.doi.org/10.1046/j.1523-1755.2002.00362.x

[24] Liu, M., Takashi, H., Morita, Y., Maruyama, S., Mizuno, M., Yuzawa, Y., et al. (2003) Non-Dipping Is a Potent Predictor of Cardiovascular Mortality and Is Associated with Autonomic Dysfunction in Haemodialysis Patients. Neph- 
rology Dialysis Transplantation, 18, 563-569. http://dx.doi.org/10.1093/ndt/18.3.563

[25] Heerspink, H., Ninomiya, T., Zoungas, S., de Zeeuw, D., Grobbee, D.E., Jardine, M.J., et al. (2009) Effect of Lowering Blood Pressure on Cardiovascular Events and Mortality in Patients on Dialysis: A Systematic Review and Meta-Analysis of Randomized Controlled Trials. The Lancet, 373, 1009-1015.

http://dx.doi.org/10.1016/S0140-6736(09)60212-9

[26] Agarwal, R. and Sinha, A.D. (2009) Cardiovascular Protection with Antihypertensive Drugs in Dialysis Patients: Systematic Review and Meta-Analysis. Hypertension, 53, 860-866. http://dx.doi.org/10.1161/HYPERTENSIONAHA.108.128116

[27] Krapf, R. and Hulter, H.N. (2009) Arterial Hypertension Induced by Erythropoietin and Erythropoiesis-Stimulating Agents (ESA). Clinical Journal of the American Society of Nephrology, 4, 470-480. http://dx.doi.org/10.2215/CJN.05040908

\section{Abbreviations}

- Blood pressure: BP

- Cardiovascular: CV

- Chronic kidney disease: CKD

- Diastolic blood pressure: DBP

- End stage renal disease: ESRD

- Erythropoiesis stimulating agents: ASE

- Hemodialysis: HD

- Left ventricular hypertrophy: LVH

- Left ventricular mass index: LVMI

- Left ventricular: LV

- National Kidney Foundation Kidney Disease Outcomes Quality Initiative: NKF-KDOQI

- New York Heart Association: NYHA

- Parathyroid hormone: PTH

- Renin-angiotensin-aldosterone system: RAAS

- Systolic blood pressure: SBP 